\title{
Laboratory scale tests for internal blast loading
}

\author{
S. Kevorkian, N. Duriez \& O. Loiseau \\ Institut de Radioprotection et Sûreté Nucléaire, France
}

\begin{abstract}
The definition of blast loads applying on a complex geometry structure is still nowadays a hard task when numerical simulation is used, essentially because of the different scales involved. As a matter of fact, modelling the detonation of a charge and its resulting load on a structure requires one to model the charge itself, the structure and the surrounding air, which rapidly leads to large size models on which parametrical studies may become unaffordable. Thus, on the basis of Crank-Hopkinson's law, an experimental set-up has been developed to support reduced scale structures as well as reduced scale detonating solid charges. As a final objective, the set-up must be used to produce the entry data for numerical assessments of the structural resistance.

The set-up is composed of two mock-ups equipped with sensors and has been designed to conduct non destructive studies. In the context of security, the general aim is to study the effects of detonation shock waves inside the test installation and to test the influence of various openings. This set-up offers the possibility of measuring the loading in terms of pressure-time curves.

The present paper summarizes the campaign of experiments performed in the year 2009 and gives the main features of the mock-up, the instrumentation and the pyrotechnics. During the campaign, internal blast tests have been conducted. Profiles of pressure versus time history are presented, taking into account relative positions of the explosive charge versus the gauges. The results obtained allow one to check that Crank-Hopkinson's law is verified and shows the gas pressure influence.
\end{abstract}

Keywords: blast waves, detonation, pressure measurements, reflections, gas pressure, safety. 


\section{Introduction}

Although important developments have taken place during the last decade, the definition of blast loads applying on a complex geometry structure is still nowadays a hard task when numerical simulation is used, essentially because of the different scales involved (both in space and time). As a matter of fact, modelling the detonation of a charge and its resulting load on a structure requires modelling the charge itself, the structure and the air surrounding the charge and the structure, which rapidly leads to large size models on which parametrical studies may become unaffordable.

Because full-scale testing of realistic target geometries and realistic effects of charge position are often prohibitively expensive and time consuming, as far as detonation is involved, small-scale testing is a well proven means to assess blast loading. The most widely used method of blast scaling is Hopkinson's "cuberoot" law for scaled distance, time and impulse.

This method has been used by IRSN to assess the pressure evolution in space in time though a free-field campaign of measurements [1]. In order to assess the pressure evolution due to small-scale detonations, IRSN realized a new campaign concerning the internal blast, which is described in this paper. Various authors used the method of blast scaling, in order to assess internal detonations. For instance, [2] shows a holographic interferometry system that permits the visualization and the measures of the propagation of an explosion of $10 \mathrm{mg}$ of silver azide cylinder inside a small-scale closed room. Reference [3] presents results of explosions of a cylindrical charge made of composition $\mathrm{B}$ explosive inside several small-scale 3 and 4-wall cubicles of different sizes and shapes, these tests were made to establish method and criteria for blast effects prediction.

Always relating to internal explosion studies, [5] presents the experimental measurements of pressure due to detonation of an explosive gaseous mixture $(1 \mathrm{~g}$ eq TNT) confined in a hemispherical soap bubble inside an unvented small-scale structure. For solid explosives, [4] presents a comparison of an experimental explosion of $1 \mathrm{lb}$ of $\mathrm{C} 4$ in a rectangular bunker and numerical calculations (Method of Images). Reference [6] shows numerical simulations done in order to study the influence of the building geometry, positions of explosion vent and ignition point. All of these studies confirm the interest of studying explosion effects at small scale, mainly allowing one to capture a better understanding of the phenomena involved.

The experimental set-up described in the present paper is a laboratory scale set-up, constituted by a mock-up, able to bear the effects of detonations of solid explosives up to $16 \mathrm{~g}$ of TNT equivalent. In the context of security, the general aim is to study the effects of blast waves inside test installations and the influence of the openings.

\section{Experimental set-up}

The experimental set-up is composed of two mock-ups and sensors; it has been designed to conduct non destructive studies. Experimental campaigns are 
performed at the SNPE's Research Centre located at Le Bouchet (Vert-le-Petit, France). SNPE ensures all the pyrotechnics handling aspects of the experiments and also provide a spare data recording system.

\subsection{Mock-ups}

For this campaign, IRSN used two mock-ups made of steel, representing a parallelepipedic room.

In order to study the scale factor in a small-scale configuration for internal blast, two mock-ups with homothetic dimensions were made. The largest mockup is $40 \mathrm{~cm}$ wide, $80 \mathrm{~cm}$ large and $40 \mathrm{~cm}$ high. The scale factor between the two different mock-ups was determined in such a way that a factor of four between the masses of explosives used in the two mock-up sizes was employed (i.e.: the scale factor $\lambda$ is equal to 0.63 for the length).

For each size, three configurations are available; the first one is the mock-up without the front face, the second one with the front face with one opening and the third one with a full front face.

Threaded holes were made through the walls, soil and roof in order to allow insertion of air blast pressure gauges. Charges are supposed to be placed at the geometrical centre of the mock-up thanks to a guiding cap inserted from the top of the box.

\subsection{Gauges}

Eleven piezoelectric pressure transducers (Kistler, reference: 603B, range: 0200 bars) are mounted on the mock-up. In the absence of pressure gauges, the holes in the walls are filled with a specific screw, so that they do not constitute unexpected venting or opening surfaces. Each pressure transducer is statically calibrated prior to the test campaign. The transducers are connected to an amplifier which is connected to the data acquisition system with electric microdot cables.
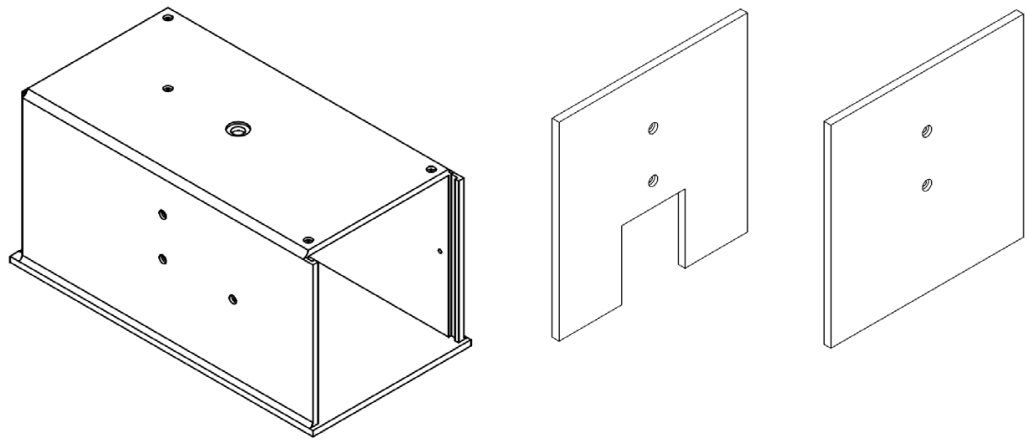

Figure 1: The mock-up, the front faces with and without openings. 


\subsection{Detonating charges}

A cylindrical charge of hexocire is initiated from one of its extremity using an electrical detonator. The masses used for the campaign described in the present paper are 1,2, 3 and $4 \mathrm{~g}$ of TNT equivalent for the smallest mock-up and 4, 8, 12 and $16 \mathrm{~g}$ of TNT equivalent for the largest one.

\subsection{Acquisition system}

A LTT-186 data acquisition and transient recorder system connected to a PC has been used for the data acquisition in parallel with a NICOLET Odyssey.

\section{Physical phenomena}

The pressures observed after the explosion of a charge in a confined space is composed of two distinct phases.

The first phase is the reflected blast load impulse and the second one is due to the pressure of the gases created during the explosion [7,8]. These phases are illustrated by Figure 2 .

\subsection{Reflections}

During an explosion, the shock wave is expanding in air up to the walls of the room, and then it is reflected (Figure 3).

For these experiments, an a priori estimate of the peak pressure was obtained using the software SHOCK [9].

In the literature, the maxima of pressure on a structure are most commonly estimated from scaled blast data or theoretical analyses of normal blast wave reflection from a rigid wall [8]. The subsequent shocks due to reflections are supposed to be attenuated.

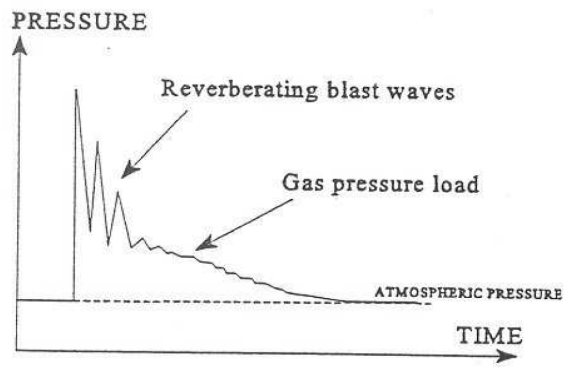

Figure 2: Profile of pressure vs. time history. 


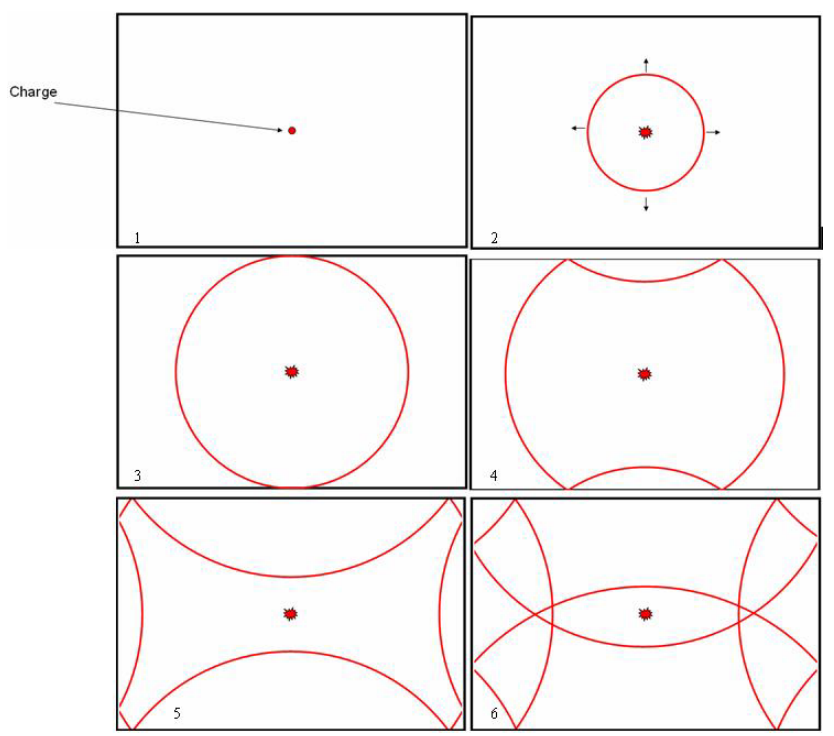

Figure 3: Air blast propagation inside a rectangular room.

\subsection{Gas pressure}

When an explosion is produced, the detonation gas generated induces an increase of pressure inside the room. This pressure decreases more or less quickly depending of the number and the size of openings in the room. This phenomenon occurs after the propagation of the air blast.

The pressure and impulse due to the detonation gases have been estimated by using the software FRANG [10].

\section{Internal blast campaign}

\subsection{Objectives}

The aim of this campaign is to validate the reduced scale experimental concept for an internal blast.

The records of pressure vs. time history are used in order to:

- Check the scaling law or Crank-Hopkinson's law in an internal blast configuration;

- Determine the characteristic parameters of the blast waves - pressure, pulse, time of arrival - identified by the time evolution of pressure and compare the values obtained with values from abacuses available in the literature or out of calculation codes $[9,10]$;

- Observe experimentally the different physical phenomena composing the air blast : shock wave with reflections and gas pressure; 
- Verify the trials reproducibility and the symmetry of measurements from symmetrically placed gauges;

- Show the difference of air blast pressure considering the position of the gauges in the room.

\subsection{Description of the trials}

For each size of the two mock-ups, four series of trials have been performed with four different masses of explosive.

For each masse of explosive, trials were performed with the three different types of opening.

For each type of opening, trials were conducted with two sets of positions for the gauges.

As a whole, 48 trials were performed, each one with eleven gauges in place. All the recordings of pressure vs. time history have been studied. Only the most representative are included in this article.

At first, the charge was placed at the exact geometrical centre of the mock-up, at the same height as the half-height gauges. By doing so, fragments of the detonator envelope came and hit the gauges after the charge initiation, causing several damages. To avoid this inconvenient, the charge was positioned at a height equal to one quarter of the overall mock-up height.

\subsection{Position of charge and gauges}

The positions of the gauges were chosen in order to verify:

- The reproducibility of the trials - gauges placed at the centre of the faces are the same for the two sets of gauges positions (K4 \& K6, K9 \& $\mathrm{K} 11)$ and two gauges of the floor are the same (K1 \& K3);

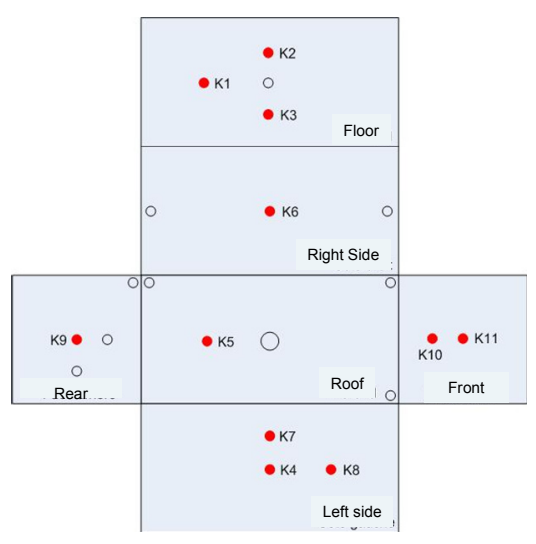

Position 1

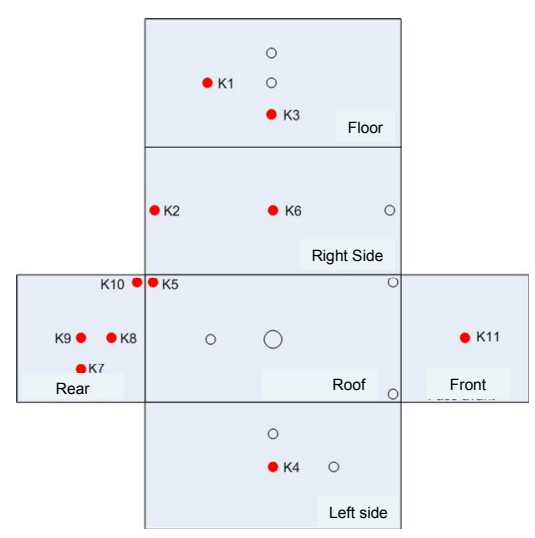

Position 2

Figure 4: Positions of the gauges for the mock-up with front face. 
- The symmetry of the propagation of the air blast - considering the different axes, pairs of symmetrical gauges were studied (K4 and $\mathrm{K} 6$, $\mathrm{K} 9$ and $\mathrm{K} 11, \mathrm{~K} 2$ and $\mathrm{K} 3, \mathrm{~K} 1$ and $\mathrm{K} 5$, for the position 1);

- The profile of pressure for the largest number of geometrical positions inside the box:

$\circ$ centre of faces, nearest from the charge (K4 and K6), farthest from the charge (K9 and K11)

$\circ$ corners (K5 and K10 for the position 2)

$\circ \quad$ edges ( $\mathrm{K} 2$ for the position 2)

$\circ \quad$ intermediate positions $(\mathrm{K} 1, \mathrm{~K} 2, \mathrm{~K} 3, \mathrm{~K} 7, \mathrm{~K} 8, \mathrm{~K} 10)$

\section{Experimental results}

Experimental results are compared with estimates obtained from SHOCK [9]. A relatively large difference has been observed between the estimates and the experimental results notably regarding the values of peak overpressure. For instance, discrepancies as large as $70 \%$ have been observed. These major discrepancies have been imputed to the shape of the explosive charge. Indeed, the SHOCK software [9] considers a spherical charge whereas the trials were performed with cylindrical charges. In the configuration used for the trials, the reduced distance between the explosive charge and the gauges is rather small (less than $\left.1 \mathrm{~m} /(\mathrm{TNT} \mathrm{kg})^{1 / 3}\right)$ and the influence of the shape of an explosive charge is more important for the smaller the distances, see Figure 5 taken from references [8] and [11]. The comparison of the impulse values between trials and a priori estimates is about $27 \%$, showing less influence of the charge shape itself.
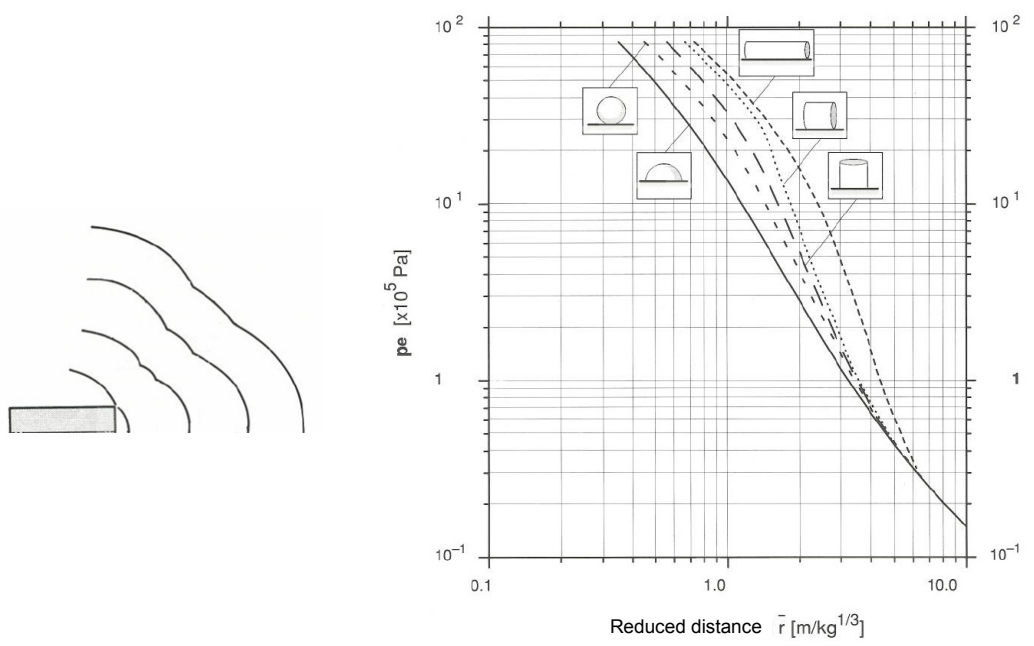

Figure 5: Influence of the shape of the charge. 


\subsection{Position of the gauges}

In this section, the different pressure profiles are presented considering the position of the gauge.

Figure 6 is the record of pressure vs. time history of the gauge K4, placed in the middle of the largest face of the experimental box. The maxima of pressure are obtained for this gauge, which is the closest gauge to the charge. It can be seen that the first overpressure peak is rather high, and followed by several peaks due to the different reflections.

Figure 7 is the record of pressure vs. time history of the gauge K9, placed in the middle of the smallest face of the experimental box. On the results, it has been observed that the largest overpressure peak appears a few microseconds after a first one, smaller, and is then followed by several peaks. This second

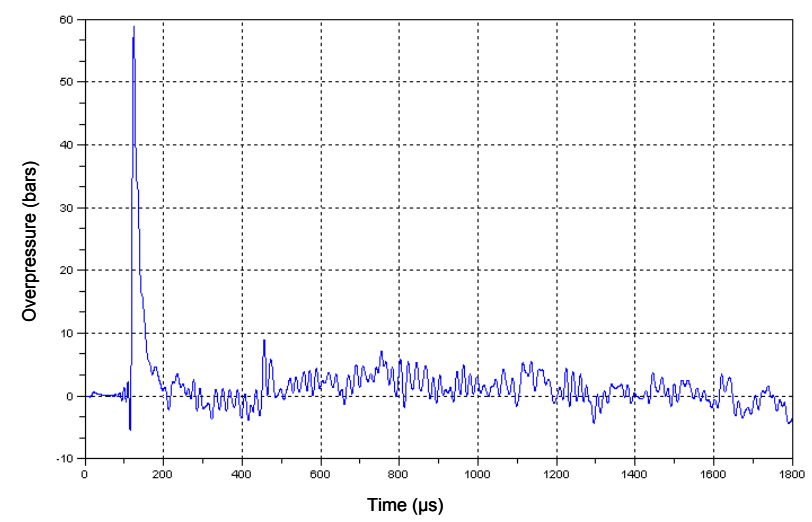

Figure 6: Centre of the largest face (K4, $16 \mathrm{~g}$ eq TNT).

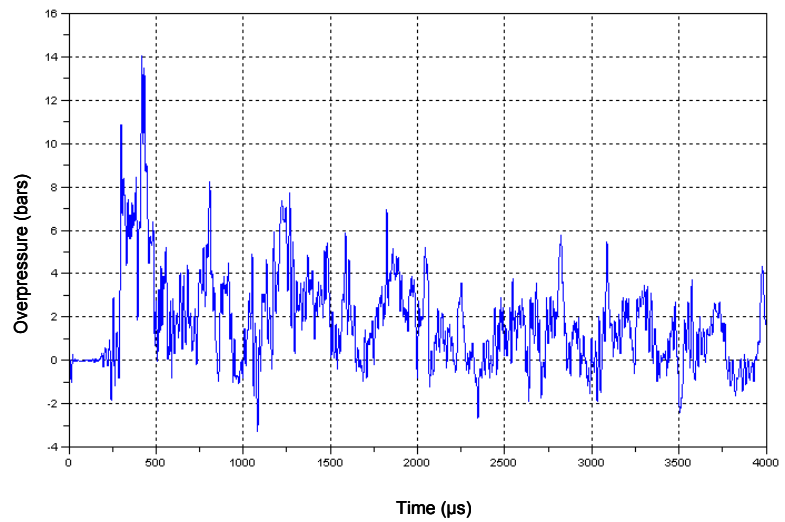

Figure 7: Centre of the smallest face (K9, $16 \mathrm{~g}$ eq TNT). 
overpressure peak is induced by the four simultaneous reflections on the walls surrounding the smallest face, which are at equal distance from the gauge.

Figure 8 is the record of pressure vs. time history of the gauge K5, placed in one corner of the room. It can be noticed that in this case, the first peak to appear is much less intense than the immediate second one. This particular gauge being near and equidistant from three surfaces of reflection, the first peak corresponds to the incident wave and the second to the combination of all the reflected waves on the adjacent faces. The following peaks are induced by the following reflections.

\subsection{Scale factor}

Considering the Crank-Hopkinson's scaling law, the pressure ratio for the two scale mock-ups should be equal to 1 , and the impulse ratio equal to the scale factor, i.e. 0.63 .

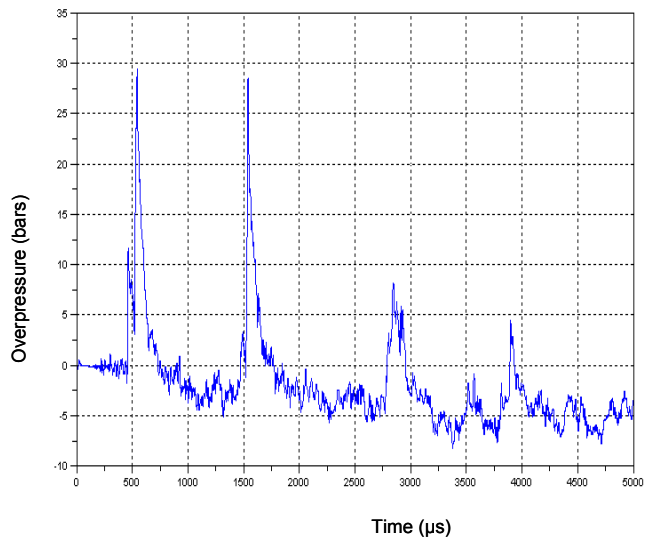

Figure 8: $\quad$ Corner (K5, 16 g eq TNT).

Table 1: Ratio between peak overpressure at two different scales. The average was obtained considering all the gauges and all the trials.

\begin{tabular}{|c|c|c|c|c|c|c|c|c|c|c|c|c|}
\hline $\begin{array}{c}\text { gauges } \\
\text { masses }\end{array}$ & K1 & K2 & K3 & K4 & K5 & K6 & K7 & K8 & K9 & K10 & K11 & Average \\
\hline $2 \mathrm{~g} / 8 \mathrm{~g}$ & 0.934 & 1.555 & 0.882 & 0.721 & 1.370 & 0.900 & 0.445 & 0.911 & 1.018 & 1.035 & 0.763 & 0.958 \\
\hline $4 \mathrm{~g} / 16 \mathrm{~g}$ & 0.636 & 1.142 & 0.677 & 1.438 & 0.957 & 0.903 & 0.591 & 0.713 & 1.510 & 0.926 & 1.044 & 0.958 \\
\hline $4 \mathrm{~g} / 16 \mathrm{~g}$ & 1.201 & 1.386 & 1.173 & 0.684 & 0.581 & 1.354 & 1.330 & 0.857 & 1.176 & 0.865 & 0.739 & 1.186 \\
\hline $4 \mathrm{~g} / 16 \mathrm{~g}$ & 1.215 & 1.104 & 0.828 & 0.629 & 0.908 & 1.145 & 0.389 & 1.179 & 1.244 & 0.738 & 0.767 & 0.922 \\
\hline $4 \mathrm{~g} / 16 \mathrm{~g}$ & 0.870 & 0.810 & 0.867 & 1.192 & 0.894 & 1.115 & 0.715 & 0.938 & 0.980 & 0.615 & 0.608 & 0.873 \\
\hline $4 \mathrm{~g} / 16 \mathrm{~g}$ & 1.306 & 0.884 & 1.445 & 0.740 & 0.730 & 1.004 & $\mathrm{x}$ & 0.763 & 0.621 & $\mathrm{x}$ & 0.870 & 0.929 \\
\hline $3 \mathrm{~g} / 12 \mathrm{~g}$ & 0.878 & 1.904 & 1.049 & 0.377 & 1.122 & 0.747 & 0.475 & 0.798 & 1.010 & 0.826 & 0.796 & 0.908 \\
\hline $3 \mathrm{~g} / 12 \mathrm{~g}$ & 0.934 & 1.530 & 0.782 & 0.599 & 1.403 & 0.645 & 0.577 & 1.077 & 0.550 & 0.693 & 1.012 & 0.891 \\
\hline $1 \mathrm{~g} / 4 \mathrm{~g}$ & 1.041 & 1.291 & 0.573 & 0.729 & 1.245 & 1.311 & 0.891 & 0.864 & 0.977 & 0.837 & 1.719 & 1.043 \\
\hline $2 \mathrm{~g} / 8 \mathrm{~g}$ & 1.180 & 0.647 & 0.875 & 0.488 & $\mathrm{x}$ & 0.477 & $\mathrm{x}$ & 0.748 & 0.845 & 0.792 & 0.754 & 0.756 \\
\hline $3 \mathrm{~g} / 12 \mathrm{~g}$ & 1.268 & 1.097 & 1.000 & 1.271 & 1.133 & $\mathrm{x}$ & 0.641 & 1.429 & 1.086 & 0.838 & $\mathrm{x}$ & 1.085 \\
\hline $4 \mathrm{~g} / 16 \mathrm{~g}$ & 0.803 & 0.942 & 0.660 & 0.816 & 1.485 & 1.602 & 0.669 & 1.128 & 0.979 & 0.576 & $\mathrm{x}$ & 0.966 \\
\hline & & & & & & & & & & Average & 0.956 \\
\hline
\end{tabular}


These results gives good insurance that the Crank-Hopkinson's scaling law has been verified through the present internal blast loading experimental campaign.

The compared recordings of the profile pressures vs. reduced time, obtained for the explosion of $4 \mathrm{~g}$ (eq TNT) in the small model and $16 \mathrm{~g}$ (eq TNT) in the large model are drawn on Figures 9 and 10. The good correspondence between the curves obtained at one scale and at another, confirms the good reproducibility of the tests even at different scales.

\subsection{Gas pressure}

For each size of mock-up and each mass of explosive, trials were performed with different type of openings in order to emphasize the influence of the gas pressure on the pressure profile measured on the mock-up faces. The recordings obtained for these three different configurations are plotted in Figure 11. During the first

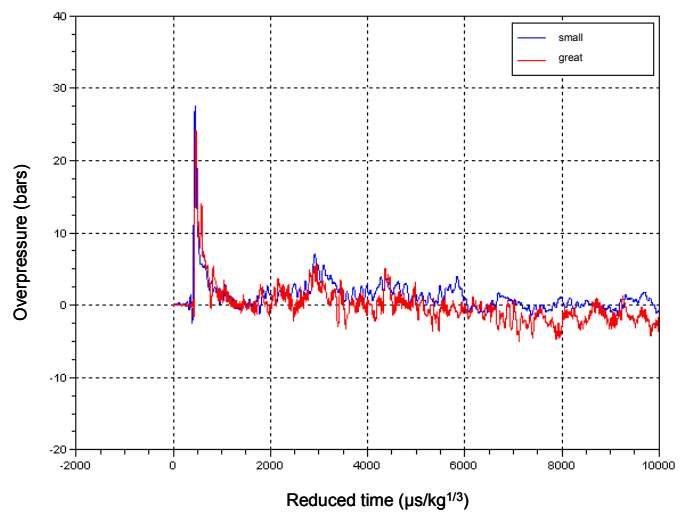

Figure 9: $\quad$ Centre of greatest face (K4, 4 and $16 \mathrm{~g}($ eq TNT)).

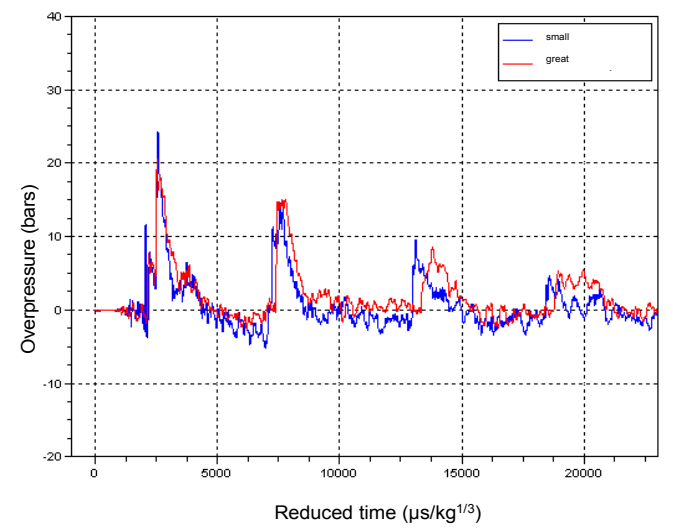

Figure 10: Corner (K5, 4 and $16 \mathrm{~g}(\mathrm{eq}$ TNT)). 


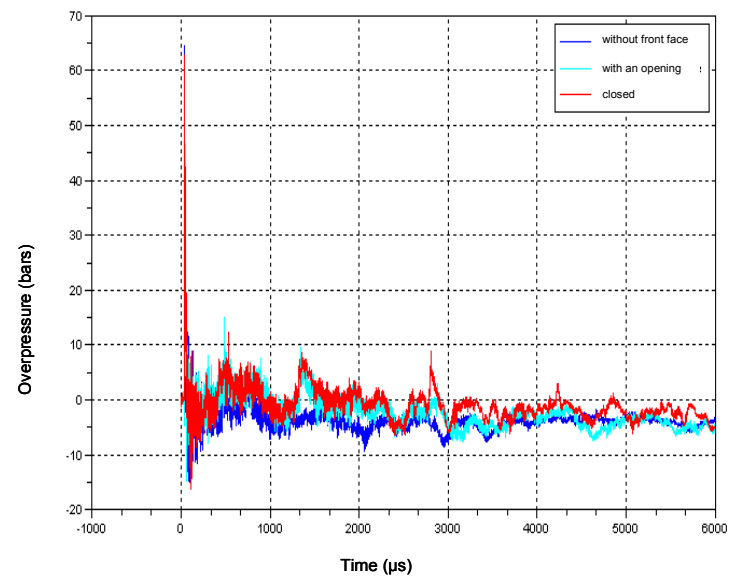

Figure 11: $\quad$ Pressure for the three type of opening (4 g eq TNT).

milliseconds of the test, the pressure profile is the same in the three configurations; this part corresponds to the air blast propagation and reflections; after that, differences between the profiles begin to appear. The pressure profiles corresponding to the closed mock-up and the mock-up with one opening present more intense peaks than in the pressure profile observed for the open mock-up. Furthermore, the amplitude of these peaks is more important in the case of closed mock-up. This observation confirms qualitatively that the detonation gas pressure has an influence on the pressures measured by the gauges. Nevertheless, considering the effect of the drift of the gauge in the $(0 ; 200$ bar) range, the evaluation of the gas pressure amplitude remains difficult to obtain out of the present experiments.

\section{Conclusions}

In order to conduct security studies for which the effects of blast waves in the vicinity of industrial sensitive installations need to be investigated, IRSN has developed an experimental set-up composed of a modular table, mock-up and pressure transducers. This experimental set-up is a support for non-destructive studies and dedicated to testing various shock wave propagations. The first campaign performed in the end of 2006 allowed to qualify the measurement chain and validate the concept of small-scale experiments. This validation was conducted through a free-field campaign. This campaign performed in 2009 allowed to validate the concept of small-scale experiments for an internal blast. In this framework, the results obtained allowed to check that the CrankHopkinson's “cube-root" law is verified. This campaign has also shown that improvements should be made in order to quantify the effects of gas pressure, notably requiring the use of some other sensor technology or range. 


\section{References}

[1] K. Cheval, O. Loiseau \& V. Vala, Laboratory scale test for the assessment of solid explosive blast effects, Structures Under Shock and Impact X,2008

[2] A; Miura, T. Mizukaki, T. Shiraisji, A. Matsuo, K. Takayama, I. Nojiri, Spread behaviour of explosion in closed space, 2004, Journal of Loss Prevention in the Process Industries 17 (2004) 81-86

[3] W.A. Keenan, J.E. Tancreto, Blast environment from fully and partially vented explosions in cubicles, 1975, Civil Engineering Laboratory

[4] P. Chan, H.H. Klein, A study of blast effects inside an enclosure, 1994, Journal of fluids engineering, 116, $\mathrm{n}^{\circ} 3,450-455$

[5] A. Zyskowski, I. Sochet, G, Mavrot, P. Bailly, J. Renard, Study of the explosion process in a small scale experiment - Structural loading, 2004, Journal of Loss Prevention in the Process Industries 17 (2004) 291-299.

[6] N. Sonoda, A. Hashimoto, A. Matsuo, Influence of vessel geometry on the effect of explosion vent, 5th International Seminar on Fire and Explosion Hazards, Edinburgh, UK, April 2007

[7] Kinney, Graham, Explosive Shocks in air, Springer Verlag (1962)

[8] Baker \& al., Explosion hazards and Evaluations, Elsevier (1983)

[9] SHOCK, Naval Civil Engineering Laboratory, USA

[10] FRANG, Naval Civil Engineering Laboratory, USA

[11] B. Anet, E. Binggli, LS2000 Luftsossphänomene infolge nuklearer und konventioneller explosionen 